\title{
Efectividad de la punción seca en el tratamiento de la fascitis plantar relacionada con las presencia de puntos gatillo miofasciales: revisión narrativa
}

Effectivity of dry needling in the treatment of plantar fasciitis related to the presence of myofascial trigger points: a narrative review

Ferrán Francés Cuscó ${ }^{1}$, Javier Alfaro Santafé ${ }^{1,2}$, Aitor Pérez Morcillo ${ }^{1,2}$, Alejandro J. Almenar Arasanz ${ }^{2}$ y Antonio Gómez Bernal ${ }^{1,2}$

'Universidad Central de Cataluña. ${ }^{2}$ Departamento de Investigación Podoactiva. Huesca, España

Palabras clave:

Punción seca, fascitis plantar, dolor de talón, dolor crónico de talón, puntos gatillo miofasciales.
Keywords:

Dry needling, plantar fasciitis, plantar heel pain, chronic heel pain, myofascial trigger points.

\section{Resumen}

Introducción: La fascitis plantar es una de las causas más comunes de dolor en el talón. La punción seca es una técnica mínimamente invasiva para tratar los puntos gatillo miofasciales. El objetivo principal de esta revisión narrativa es describir la efectividad de la punción seca en el tratamiento de la fascitis plantar.

Material y métodos: Búsqueda bibliográfica en cuatro bases de datos (PubMed, Cochrane, Dialnet y Researchgate). Se tuvieron en cuenta los estudios de los últimos 6 años (desde 2014 hasta 2019) que hablaran de la técnica de punción seca y el tratamiento de la fascitis plantar. Los términos de búsqueda fueron: "Dry needling", "plantar fasciitis", "plantar heel pain", "chronic heel pain" y "myofascial trigger points".

Resultados: Se revisaron un total de 7 artículos a texto completo de los últimos 6 años, donde se analizaron un total de 395 pacientes.

Conclusiones: La punción seca parece ser una buena técnica para la disminución del dolor y la mejoría de la funcionalidad del pie afectado por la fascitis plantar a corto y largo plazo.

\section{Abstract}

Introduction: Plantar fasciitis is one of the most common causes of heel pain. Dry needling is a minimally invasive technique to treat myofascial trigger points. The main objective of this narrative review is to describe the effectiveness of dry needling in the treatment of plantar fasciitis.

Material and methods: Four databases were used to carry out a bibliography search (PubMed, Cochrane, Dialnet and Researchgate). Studies from the previous 6 years (between 2014 and 2019) that mention dry needling technique and the treatment of plantar fasciitis were taken into account. The following terms were used: "Dry needling", "plantar fasciitis", "plantar heel pain", "chronic heel pain and myofascial trigger points".

Results: A total of 7 full-text articles from the last 6 years were reviewed. A total of 395 patients were analyzed

Conclusion: Dry needling seems to be a good technique for reducing pain and improving the functionality of the foot affected by plantar fasciitis in the short and long term. 


\section{INTRODUCCIÓN}

La fascia plantar es una amplia banda de tejido conectivo que soporta el arco del pie, la cual está compuesta por un componente central grueso y dos componentes más, uno lateral y otro medial ${ }^{1}$. Esta fascia se origina proximalmente en el tubérculo medial del calcáneo, y se extiende dividiéndose de manera distal en cinco bandas que se insertan en la base de las falanges proximales de cada dedo ${ }^{1,2}$. Esta estructura genera el mecanismo de Windlass. Es un término usado para describir cómo actúa la aponeurosis plantar, dando tensión durante la dorsiflexión de los dedos y estabilizando/ elevando el arco medial².

La fascia plantar puede convertirse en una degeneración patológica llamada fascitis plantar (FP), una de las causas más comunes de dolor en el talón ${ }^{2,3}$. Se estima que una de cada diez personas desarrollarán dolor en el talón a lo largo de su vida, relacionando un $80 \%$ de los casos a las dolencias producidas por la fascitis plantar 4 .

El pico de incidencia de las FP se encuentra en personas de mediana y avanzada edad, siendo más común en mujeres $^{5}$. También afecta a un $10 \%$ de los corredores y es común en trabajadores y atletas, los cuales, en las actividades que realizan, tienen un gran impacto en el pie $^{3}$. La FP es causada por factores que producen una sobrecarga en la inserción de la fascia plantar del calcáneo causando microrroturas de esta $^{6,7}$.

Cuanto antes se diagnostique y se inicie el tratamiento, mayor es la probabilidad de éxito. El diagnóstico se realiza mediante la historia clínica y la exploración física ${ }^{5}$. Las modalidades diagnósticas por imagen se utilizarán cuando la historia clínica y la evaluación clínica no sean concluyentes ${ }^{3}$.

Alrededor de un $90 \%$ de los pacientes son tratados con tratamientos conservadores como primera opción ${ }^{8}$. Los tratamientos conservadores más comunes son: reposo, ejercicios, estiramientos o medicación antinflamatoria ${ }^{3,8}$. Si en un plazo de unos 6 meses los síntomas no mejoran, entonces se propondría la intervención quirúrgica4

El síndrome de dolor miofascial (SDM) tiene como característica la presencia de puntos gatillo miofasciales (PGM). Se define un PGM como un nódulo hipersensible en una banda tensa del músculo esquelético cuya estimulación (palpación, compresión, contracción o estiramiento) produce dolor local $\left.\right|^{9,10}$. Los signos más evidentes en la presencia de PGM se perciben a la palpación ${ }^{11}$. El primer signo es localizar un nódulo doloroso dentro de una banda tensa, la cual implica a un cierto número de fibras musculares con una rigidez incrementada ${ }^{12}$. La palpación perpendicular o la punción de un PGM suelen dar lugar a lo que se denomina respuesta de espasmo local (REL), que se define como una contracción súbita, momentánea y espontánea de las fibras musculares que pertenecen a una banda tensa ${ }^{13}$. El dolor referido también es un signo en los PGM, el cual se percibe en una región distinta y/o alejada de la zona primaria de la lesión variando su extensión ${ }^{12}$.
En cuanto a la sintomatología de los PGM, esta es muy diversa, dependiendo de la percepción de cada individuo en las características del dolor (punzante, irritante...) intensidad, distribución y duración. Esta percepción tan individualizada hace que sea un reto el poder estandarizar y validar estos síntomas para un claro diagnóstico ${ }^{9}$.

El tratamiento se concentra en la terapia física, donde el objetivo es reducir el dolor y restaurar la funcionalidad normal del músculo ${ }^{12}$. La mayoría de los tratamientos tienen como objetivo desactivar los PGM ${ }^{14}$. La presencia de PGM en la musculatura del pie y de la pierna tiene un rol importante en el dolor del talón que refieren los pacientes con fascitis plantar ${ }^{15}$.

La punción seca (PS) se define como una técnica mínimamente invasiva utilizada por terapeutas físicos para tratar el SDM a través de una aguja delgada, sin inyectar medicación. Estas se insertan en áreas de músculo conocidos como PGM ${ }^{10,16,17}$. En la bibliografía actual se observan diversas modalidades de PS para el tratamiento de los PGM. Estas se pueden clasificar según diferentes criterios, pero el más utilizado es el que valora la profundidad a la que alcanza la aguja, atravesando los PGM o quedando en los tejidos subyacentes a este en-20. $^{18}$.

Dependiendo de si la PS es superficial o profunda, obtendremos diversos beneficios. En la PS superficial se conseguirá un efecto analgésico debido a la estimulación de fibras nerviosas A-delta y A-beta. En la PS profunda encontraríamos los mecanismos involucrados en la PS superficial debido que al pinchar los tejidos suprayacentes también estamos realizando una PS superficial. A parte de estos mecanismos le podemos sumar: lavado de sustancias sensibilizantes producido por las REL, elevación del pH, laceración mecánica de los miocitos o de las placas motoras afectadas, efectos beneficiosos sobre el flujo sanguíneo y la analgesia mediada por la inflamación provocada ${ }^{18}$.

El objetivo de la presente revisión narrativa es el de analizar los efectos de la PS en los cambios de la funcionalidad del pie afectado, detallar los efectos de la PS en los cambios de dolor percibidos por el paciente, identificar los efectos de la PS en los cambios de la calidad de vida, describir el tipo de aguja empleado para la PS y mencionar la musculatura tratada para la FP.

\section{MATERIAL Y MÉTODOS}

Para la obtención de esta revisión narrativa se realizó una búsqueda bibliográfica donde se consultaron las siguientes bases de datos: PubMed, Cochrane, Dialnet y Researchgate. Para la selección de los artículos se siguieron las recomendaciones de la metodología PRISMA ${ }^{21,22}$. Las palabras clave utilizadas en la búsqueda de artículos relacionados con la efectividad de la PS en el tratamiento de la FP fueron "Dry needling AND plantar fasciitis", "Dry needling AND plantar heel pain", "Dry needling AND chronic heel pain" y Dry needling AND plantar fasciitis AND myofascial trigger points" (Tabla I). 


\begin{tabular}{|c|c|c|c|c|}
\hline & PubMed & Cochrane & Dialnet & Researchgate \\
\hline Dry needling AND plantar fasciitis & 26 & 22 & 0 & 52 \\
\hline Dry needling AND plantar heel pain & 14 & 12 & 0 & 61 \\
\hline Dry needling AND chronic heel pain & 2 & 1 & 0 & 78 \\
\hline Dry needling AND plantar fasciitis AND myofascial trigger points & 8 & 4 & 0 & 10 \\
\hline
\end{tabular}

En un primer momento se recuperaron un total de 290 artículos que respondían a los términos de búsqueda usando las combinaciones de palabras clave nombradas anteriormente; 228 fueron excluidos por el título y el resumen por no tener relación directa con el tema, 10 por duplicidad y 27 por fechas de publicación, escogiendo publicaciones recientes desde 2014 hasta 2019. De los 25 artículos restantes, tras la aplicación de los criterios de inclusión y de exclusión, quedaron un total de 7 artículos para el análisis completo de sus resultados (Figura 1).

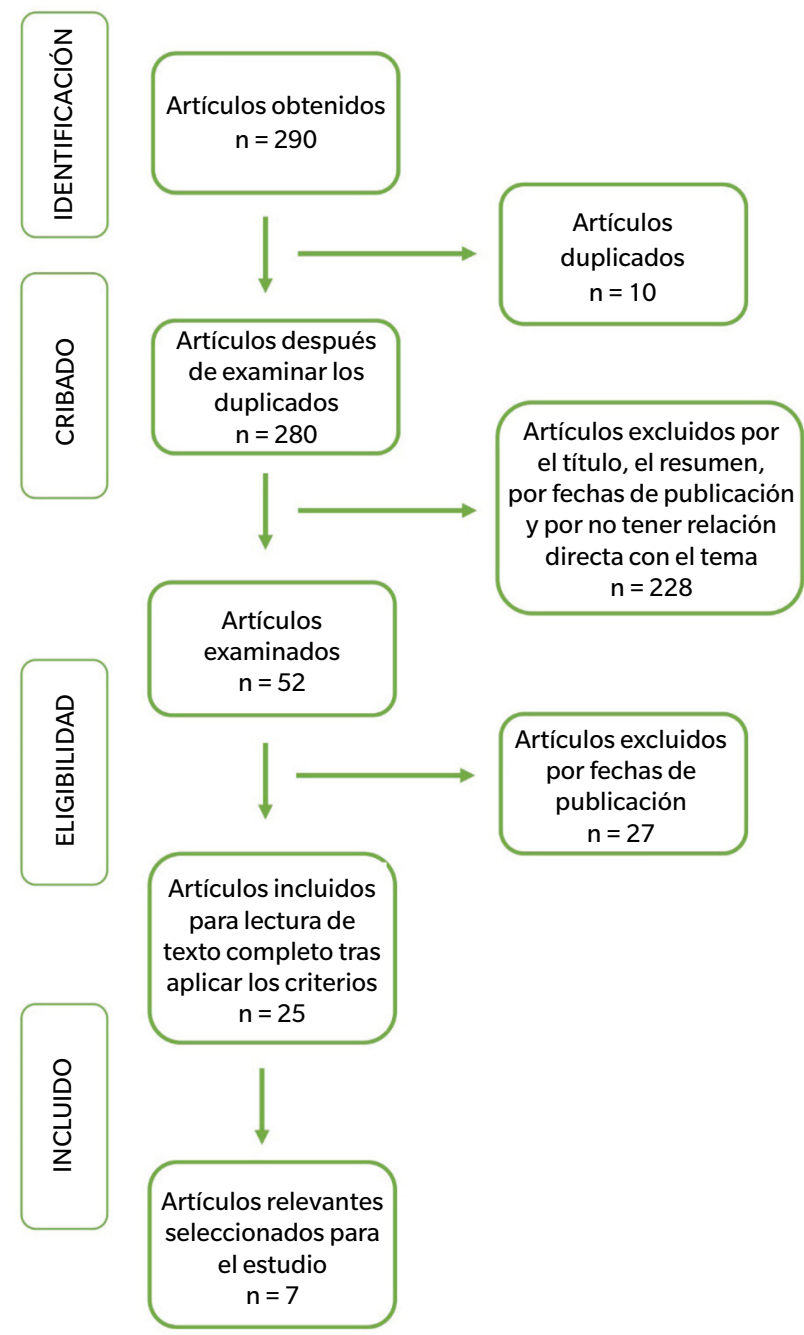

Figura 1. Diagrama de flujo basado en las recomendaciones del método PRISMA para la selección de artículos ${ }^{21,22}$.
Los criterios de inclusión fueron: punción seca utilizada para el tratamiento de la fascitis plantar, muestra con dolor en la zona del talón, edad de la muestra comprendida entre los $18 \mathrm{y}$ 80 años. Los criterios de exclusión fueron: revisión sistemática, acupuntura utilizada para el tratamiento de los puntos gatillo miofasciales, publicaciones anteriores al año 2014 valorando los artículos más actuales de los últimos 6 años.

Tras la aplicación de los criterios, esta revisión narrativa se ha basado en 7 artículos para el análisis completo de sus resultados. Todos ellos corresponden a los 6 últimos años (desde 2014 hasta 2019) y todos fueron publicados en inglés.

Los artículos seleccionados fueron clasificados siguiendo los niveles de evidencia según la escala de United States Preventive Servies Task Force (USPSTF) ${ }^{23}$.

\section{RESULTADOS}

Se analizaron un total de 7 artículos, todos ellos de los 6 últimos años. Según la escala USPSTF ${ }^{23}$ Ios niveles de evidencia en todos ellos fue $\mathrm{I}^{24-30}$ donde un total de 395 pacientes fueron analizados (Tabla II).

En la Tabla III se muestran además del autor, el año de publicación, las características de la muestra, la duración del tratamiento, las evaluaciones, la duración de la FP, el tipo de aguja y la localización de la aguja de cada artículo.

En la mayoría de los artículos se evalúa la función del pie $25-27,29,30$ y en los mismos u otros también miden la intensidad del dolor en el pie 24,25,27-29. En algunos artículos se evalúan otros parámetros, como es en el caso de Mathheu y cols. ${ }^{24}$ que evalúan la calidad de vida de los pacientes y Bina y cols. ${ }^{25}$ que evalúan el rango de movilidad del tobillo en dorsiflexión (Tabla IV).

La duración del tratamiento varía en los diversos artículos, siendo de 1 semana ${ }^{28}, 3$ semanas $^{29,30}, 4$ semanas $^{25,27}$ y de 6 semanas $^{24,26}$. El tiempo de evolución de la FP también es diferente en los artículos, siendo superior a un $\operatorname{mes}^{24-26,28} \mathrm{O}$ superior a los tres meses $27,28,30$ (Tabla III).

Respecto la localización de la aguja, varios artículos coinciden en una única zona como es la planta del pie ${ }^{28-30}$, un único artículo se centra en los gemelos ${ }^{25}$ y el resto lo aplica en una gran diversidad de músculos según cada caso específico ${ }^{24,26,27}$. Se encuentran diferentes parámetros en prácticamente todos los estudios referentes al número de evaluaciones y el tipo de agujas empleadas (Tabla III). 
Tabla II. Resumen de los artículos incluidos y clasificación según niveles de calidad de la evidencia (USPSTGF) ${ }^{23}$.

\begin{tabular}{|c|c|c|c|c|}
\hline Autor & Año & Nivel de evidencia & Muestra pacientes & Rango de edad \\
\hline Matthew y cols. ${ }^{24}$ & 2014 & I & 79 & +18 \\
\hline Bina y cols. $^{25}$ & 2016 & I & 20 & +18 \\
\hline Reem y cols. ${ }^{26}$ & 2017 & I & 30 & +18 \\
\hline Andrew y cols. ${ }^{27}$ & 2017 & I & 30 & $18-55$ \\
\hline Shirvan y cols. ${ }^{28}$ & 2017 & I & 66 (38 M y $28 \mathrm{H})$ & +18 \\
\hline Mohammad y cols. ${ }^{29}$ & 2018 & I & 72 & $23-65$ \\
\hline Esat y cols. ${ }^{30}$ & 2019 & I & 98 & $26-73$ \\
\hline
\end{tabular}

\section{Tabla III. Características de interés de los artículos a analizar.}

\begin{tabular}{|c|c|c|c|c|c|c|}
\hline Autor (año) & $\begin{array}{l}\text { Características de la } \\
\text { muestra }\end{array}$ & $\begin{array}{l}\text { Duración del } \\
\text { tratamiento }\end{array}$ & Evaluaciones & $\begin{array}{l}\text { Duración } \\
\text { de la FP }\end{array}$ & Tipo de aguja & Localización de la aguja \\
\hline $\begin{array}{l}\text { Matthew y cols. } \\
(2014)^{24}\end{array}$ & $\begin{array}{l}\text { N: } 79 p \\
\text { Gc: } 38 p \\
\text { Ge: } 41 p \\
\text { Edad media: } 56.1 \text { años }\end{array}$ & 6 semanas & $\begin{array}{l}\text { Principio, } 2,4, \\
6 \text { y } 12 \text { semanas }\end{array}$ & $>1 \mathrm{mes}$ & $30-75 \times 0.3 \mathrm{~mm}$ & $\begin{array}{l}\text { Sóleo, cuadrado plantar, } \\
\text { flexor común y abductor del } \\
\text { hallux. Otros músculos pero } \\
\text { de forma secundaria }\end{array}$ \\
\hline $\begin{array}{l}\text { Bina y cols. } \\
(2016)^{25}\end{array}$ & $\begin{array}{l}\text { N: } 20 p \\
\text { Gc: } 10 p \\
\text { Ge: } 10 p \\
\text { Edad media: } 50.6 \text { años }\end{array}$ & 4 semanas & $\begin{array}{l}\text { Principio, } 4 \text { y } 8 \\
\text { semanas }\end{array}$ & $>1$ mes & $30-50 \times 0.6 \mathrm{~mm}$ & Gemelos \\
\hline $\begin{array}{l}\text { Reem y cols. } \\
(2017)^{26}\end{array}$ & $\begin{array}{l}\text { N: } 30 p \\
\text { Gc: } 15 p \\
\text { Ge: } 15 p \\
\text { Edad media: } 44 \text { años }\end{array}$ & 6 semanas & $\begin{array}{l}\text { Principio, } 6 \text { y } 12 \\
\text { semanas }\end{array}$ & $>1 \mathrm{mes}$ & $30-75 \times 0.3 \mathrm{~mm}$ & $\begin{array}{l}\text { Tuberosidad medial } \\
\text { del calcáneo, tendón } \\
\text { de Aquiles, gemelos, } \\
\text { sóleo, bíceps femoral y } \\
\text { semimembranoso }\end{array}$ \\
\hline $\begin{array}{l}\text { Andrew y cols. } \\
(2017)^{27}\end{array}$ & $\begin{array}{l}\text { N: } 30 \mathrm{p} \\
\text { Gc: } 15 \mathrm{p} \\
\text { Ge: } 15 \mathrm{p} \\
\text { Edad media: } 46 \text { años }\end{array}$ & 4 semanas & $\begin{array}{l}\text { Principio y } 4 \\
\text { semanas }\end{array}$ & $>3$ meses & $30-50 \times 0.25 \mathrm{~mm}$ & $\begin{array}{l}\text { Sóleo, flexor común, } \\
\text { abductor del halluxy } \\
\text { gemelos }\end{array}$ \\
\hline $\begin{array}{l}\text { Shirvan y cols. } \\
(2017)^{28}\end{array}$ & $\begin{array}{l}\text { N: } 66 \mathrm{p} \\
\text { Gc: } 32 \mathrm{p} \\
\text { Ge: } 34 \mathrm{p} \\
\text { Edad media: } 41 \text { años }\end{array}$ & 1 semana & $\begin{array}{l}\text { Principio, } 3 \text { y } 6 \\
\text { semanas, } 3 \text { y } 6 \\
\text { meses y } 1 \text { año }\end{array}$ & $>3$ meses & $50 \times 0.3 \mathrm{~mm}$ & Planta del pie \\
\hline $\begin{array}{l}\text { Mohammad y } \\
\text { cols. }(2018)^{29}\end{array}$ & $\begin{array}{l}\text { N: } 72 \mathrm{p} \\
\text { Gc: } 36 \mathrm{p} \\
\text { Ge: } 36 \mathrm{p} \\
\text { Edad media: } 44 \text { años }\end{array}$ & 3 semanas & $\begin{array}{l}\text { Principio, } 4 \text { y } 8 \\
\text { semanas }\end{array}$ & $>1 \mathrm{mes}$ & $10 \mathrm{~mm}$ & Región medial del pie \\
\hline $\begin{array}{l}\text { Esat y cols. } \\
(2019)^{30}\end{array}$ & $\begin{array}{l}\text { N: } 98 p \\
\text { Gc: } 49 p \\
\text { Ge: } 47 p \\
\text { Edad media: } 49 \text { años }\end{array}$ & 3 semanas & $\begin{array}{l}\text { Principio, } 3 \\
\text { semanas y } 6 \\
\text { meses }\end{array}$ & $>3$ meses & $40 \times 0.25 \mathrm{~mm}$ & Planta del pie \\
\hline
\end{tabular}

N: Muestra. Gc: grupo control. Ge: grupo experimental. mm: milímetros.

Finalmente, todos los artículos muestran un beneficio de la PS en los resultados de funcionalidad y dolor en el pie de los test evaluados $^{24-30}$. De los artículos mencionados, dos test no encuentran diferencias significativas en sus resultados. Es el caso de Mattheu y cols. ${ }^{24}$ respecto la calidad de vida, y Bina y cols. ${ }^{25}$ refiriéndose al rango de movilidad del tobillo en dorsiflexión (Tabla IV). 


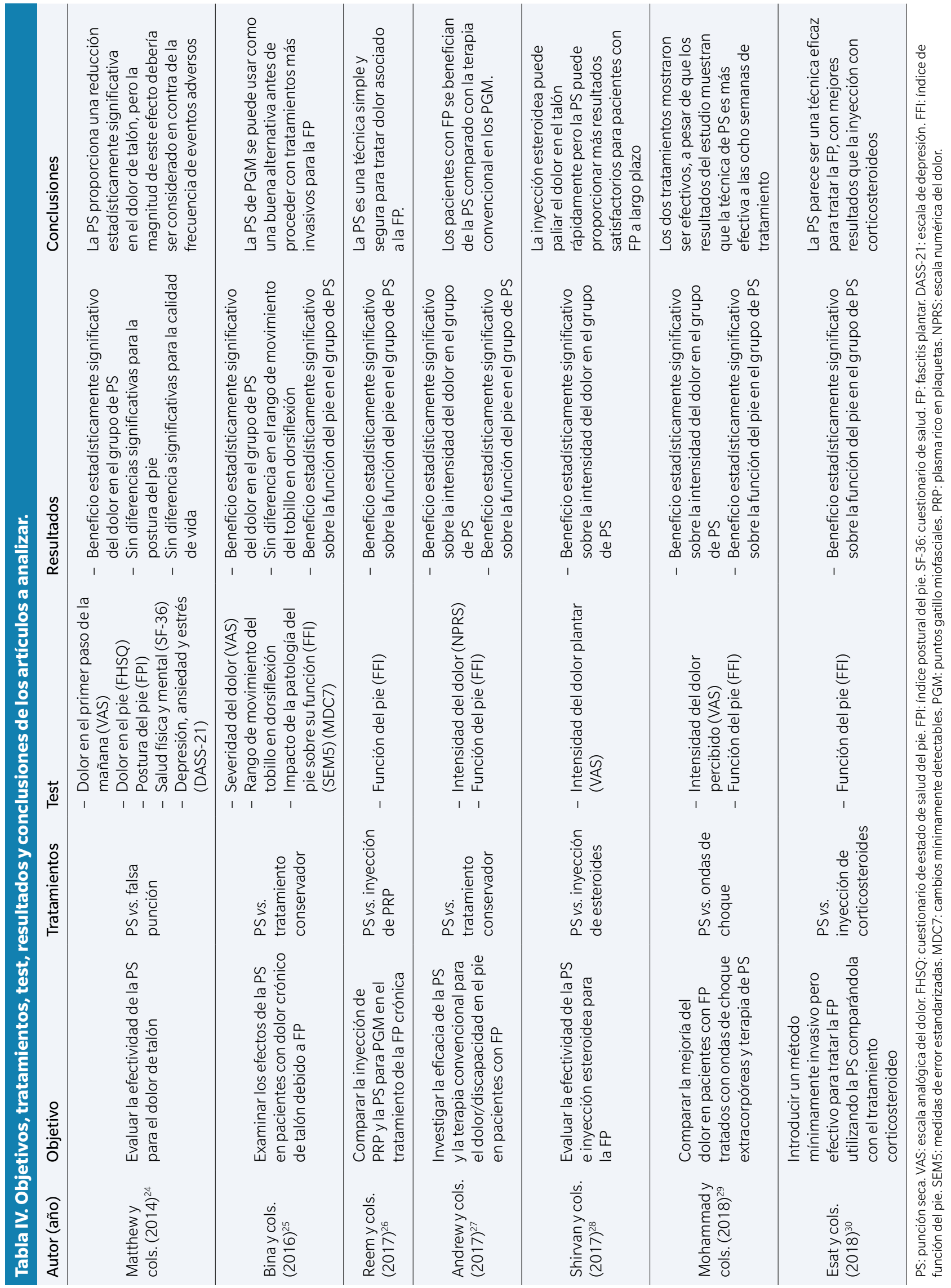




\section{DISCUSIÓN}

Cinco artículos valoran la funcionalidad del pie, cuatro de ellos lo hacen con la escala $\mathrm{FFI}^{26,27,29,30}$ y uno a través de SEM5 y MDC $7^{25}$. Mohammad y cols. ${ }^{29}$ y Esat y cols. ${ }^{30}$ ofrecen un tratamiento de 4 semanas de duración, donde se encuentra un descenso de 41.9 puntos y 33.3 puntos en la escala $\mathrm{FFI}$, respectivamente. La menor reducción de Esat y cols. ${ }^{30}$ se podría atribuir al hecho que sobrestimula la zona realizando dos sesiones de tratamiento la misma semana respecto Mohammad y cols. ${ }^{29}$, que solo procede con una sesión de tratamiento. Andrew y cols. ${ }^{27}$ y Mohammad y cols. ${ }^{29}$ realizan una evaluación en la cuarta semana, obteniendo una reducción en la puntuación de 18.46 y 41.9 puntos, respectivamente. Este dato nos podría indicar la importancia de la actuación en la musculatura específica del pie, ya que Andrew y cols. ${ }^{27}$ actúa en cuatro músculos diferentes (sóleo, flexor común, abductor del hallux y gemelos) mientras que Mohammad y cols. ${ }^{29}$ solo actúa en la planta del pie. Todos los artículos que valoran la funcionalidad del pie para el tratamiento de la FP a través de la PS comparada con otras técnicas observan un beneficio estadísticamente significativo ${ }^{25-27,29,30}$. Encontramos un dato interesante que comentan Mohammad y cols. ${ }^{29}$ y Esat y cols. ${ }^{30}$ donde sus evaluaciones se desarrollan a largo plazo, mostrando una efectividad duradera en el tiempo que sería beneficiosa antes de actuar con técnicas más invasivas. Esta premisa no se confirma con el estudio de Reem y cols. ${ }^{26}$, el cual solo observa esta diferencia en el dolor percibido por el paciente, no en la funcionalidad. Los estudios de Bina y cols. ${ }^{25}$ y Andrew y cols. ${ }^{27}$, que comparan la PS con tratamiento conservador, no mencionan la premisa anteriormente comentada.

Tal como se muestra en los resultados, los artículos que solo valoran el dolor del pie afectado lo hacen con la escala de valoración VAS ${ }^{25,28,29}$, menos uno que utiliza la NPRS27 y Matthew y cols. ${ }^{24}$, que además de utilizar la escala VAS para evaluar el dolor del primer paso, añade la escala FHSQ. Bina y cols. ${ }^{25}$, Andrew y cols. ${ }^{27}$ y Mohammad y cols. ${ }^{29}$ hacen una evaluación en la cuarta semana consiguiendo una disminución de 5.6 puntos, 2.2 puntos y 3.5 puntos, respectivamente. La mejora superior en el estudio de Bina y cols. ${ }^{25}$ podría deberse a la duración de tratamiento de 4 semanas, respecto al de 3 semanas de Mohammad y cols. ${ }^{29}$. La diferencia numérica de entre los tres artículos ${ }^{25,27,29}$ podría deberse a que la PS se centra en diferentes músculos.

Todos los artículos, independientemente del test, muestran resultados estadísticamente significativos respecto el tratamiento de la FP a través de la PS, y también coinciden que este efecto se prolonga a largo plazo $24,25,27-29$. Estos resultados muestran una línea de actuación esperanzadora para poder tratar la FP con PS a largo plazo con resultados beneficiosos.

Respecto la calidad de vida, tan solo un artículo lo evalúa y sin encontrar resultados estadísticamente significativos ${ }^{24}$. Esta variable queda poco definida debido a que los otros autores no la han valorado, dando más hincapié al dolor y a la función del pie afectado.
A la hora de tratar los músculos afectados hubo discrepancias en las localizaciones, ya que tres artículos lo hacen solo

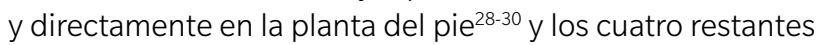
lo hacen a través de diversos músculos de la parte inferior de la pierna y pie $\mathrm{e}^{24-27}$. A pesar de la gran disparidad de músculos tratados en los estudios, todos ellos consiguen resultados beneficiosos, aportando relajación del músculo al reducir la tensión en este. Aun así, sería importante la unificación en criterio a la hora de tratar los músculos para la FP. Se observa una relación en los músculos tratados y el tipo de aguja escogida, utilizando las de menor longitud en los estudios

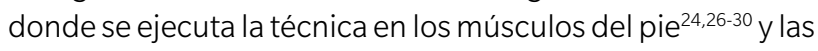
agujas de mayor longitud para los diferentes músculos que no se encuentren en el pie $\mathrm{e}^{24-27}$. Estos factores se ven modificados por la profundidad en la que se encuentra el músculo y la técnica utilizada en cada uno de ellos. Sería de gran valor poder estandarizar las agujas para cada músculo para poder elaborar un protocolo de actuación para el tratamiento de la FP a través de la PS.

No hay un consenso en la duración que tiene que tener el tratamiento de la FP a través de la PS, ya que Mohammad y cols. ${ }^{29}$ y Esat y cols. ${ }^{30}$ lo hacen de 3 semanas, Bina y cols. ${ }^{25}$ y Andrew y cols. ${ }^{27}$ de 4 semanas, y finalmente Mathhew y cols. ${ }^{24}$ y Reem y cols. ${ }^{26}$ de 6 semanas. Shirvan y cols. ${ }^{28}$ no menciona la duración del tratamiento. Respecto el número de sesiones aplicadas en cada semana, hay mayor coincidencia entre autores, siendo de una sola aplicación a la semana en casi todos ${ }^{24-29}$, menos en uno, Esat y cols. ${ }^{30}$ que lo aplica dos veces a la semana sin obtener datos relevantes comparado con el resto de artículos. A pesar de todas estas variaciones, en los siete artículos consultados los resultados respecto el beneficio de la PS son estadísticamente significativos en cuanto a las valoraciones pre y postratamiento ${ }^{24-30}$.

Un último aspecto a valorar es el de los efectos adversos producidos por la PS. Los artículos de Reem y cols. ${ }^{26}$, Andrew y cols. ${ }^{27}$ y Shirvan y cols. ${ }^{28}$ no comentan nada de los efectos adversos, pero otros artículos sí lo hacen ${ }^{24,25,29,30}$. Bina y cols. ${ }^{25}$ menciona que estos son efectos mínimos y otros autores, como Mathhew y cols. ${ }^{24}$, Mohammad y cols. ${ }^{29}$ y Esat y cols. ${ }^{30}$ los describen como ligeros hematomas y pequeña exacerbación del dolor pospunción. Por lo tanto, la PS es considerada como una técnica óptima para el tratamiento efectivo de la FP sin casi efectos adversos.

Hay que tener en cuenta ciertas limitaciones en esta revisión narrativa, como la dificultad en agrupar y unificar el sexo de los individuos y también el tamaño de las muestras, ya que con un número mayor se podrían obtener resultados más descriptivos.

En futuros estudios, se hace necesario seguir investigando la técnica para poder unificar criterios para la realización de la PS tanto en duración de tratamiento como en material y músculos tratados. Estudios más amplios y con mayores muestras serán necesarios para poder dar lugar a premisas más concluyentes para la mejora de la salud y bienestar de los pacientes a través de la PS. 


\section{CONCLUSIÓN}

Los resultados del presente trabajo sugieren que la punción seca mejora de forma significativa la funcionalidad del pie y el dolor derivado de la fascitis plantar, mostrando mayor eficacia cuando se trata en específico la musculatura del pie. Es importante aplicar la técnica una vez a la semana para conseguir el mayor beneficio y así no alcanzar la sobrestimulación. A través de esta revisión narrativa es posible concluir que la punción seca podría una técnica eficaz para tratar la fascitis plantar a corto y largo plazo.

\section{BIBLIOGRAFÍA}

1. Cutts S, Obi N, Pasapula C, Chan W. Plantar fasciitis. Ann R Coll Surg Engl. 2012;94(8):539-42. DOI: 10.1308/003588412X131712215924 56.

2. Huffer D, Hing W, Newton R, Clair M. Strength training for plantar fasciitis and the intrinsic foot musculature: A systematic review. Phys Ther Sport. 2017;24:44-52. DOI: 10.1016/j.ptsp.2016.08.008.

3. Stecco C, Corradin M, Macchi V, Morra A, Porzionato A, Biz C, et al. Plantar fascia anatomy and its relationship with Achilles tendon and paratenon. J Anat. 2013;223(6):665-76. DOI: 10.1111/joa.12111.

4. Rosenbaum AJ, DiPreta JA, Misener D. Plantar Heel Pain. Med Clin North Am. 2014;98(2):339.52. DOI: 10.1016/j.mcna.2013.10.009.

5. Thompson J, Saini SS, Reb CW, Daniel JN. Diagnosis and Management of Plantar Fasciitis. J Am Osteopath Assoc. 2014;114(12):900-6. DOI: 10.7556/jaoa.2014.177.

6. Orchard J. Plantar fasciitis. BMJ. 2012;345(oct10 1):e6603. DOI: 10.1136/bmj.e6603..

7. Sung KC, Chung JY, Feng IJ, Yang SH, Hsu C-, Lin HJ, et al. Plantar fasciitis in physicians and nurses: a nationwide population-based study. Ind Health. 2019;58(2):153-60. DOI: 10.2486/indhealth.2019-0069.

8. Giordani F, Bernini A, Müller-Ehrenberg H, Stecco C, Masiero S. A global approach for plantar fasciitis with extracorporeal shockwaves treatment. Eur J TransI Myol. 2019;29(3):8372. DOI: 10.4081/ ejtm.2019.8372.

9. Shah JP, Thaker N, Heimur J, Aredo JV, Sikdar S, Gerber L. Myofascial Trigger Points Then and Now: A Historical and Scientific Perspective. PM\&R. 2015;7(7):746-61. DOI: 10.1016/j.pmrj.2015.01.024.

10. Espejo-Antúnez L, TejedaJFH, Albornoz-Cabello M, Rodríguez-Mansilla $\mathrm{J}$, de la Cruz-Torres B, Ribeiro F, et al. Dry needling in the management of myofascial trigger points: A systematic review of randomized controlled trials. Complement Ther Med. 2017;33:46-57. DOI: 10.1016/j. ctim.2017.06.003.

11. Moraska AF, Schmiege SJ, Mann JD, Butryn N, KrutschJP. Responsiveness of Myofascial Trigger Points to Single and Multiple Trigger Point Release Massages: A Randomized, Placebo Controlled Trial. Am J Phys Med Rehabil. 2017;96:639-45. DOI: 10.1097/PHM.0000000000000728.

12. Barbero M, Schneebeli A, Koetsier E, Maino P. Myofascial pain syndrome and trigger points: evaluation and treatment in patients with musculoskeletal pain. Curr Opin Support Palliat Care. 2019;13(3):2706. DOI: 10.1097/SPC.0000000000000445.

13. Gerwin RD. Diagnosis of myofascial pain syndrome. Phys Med Rehabil Clin N Am. 2014;25(2):341-55. DOI: 10.1016/j.pmr.2014.01.012.
14. Gerwin RD. Myofascial Trigger Point Pain Syndromes. Semin Neurol 2016;36(5):469-73. DOI: 10.1055/s-0036-1586262.

15. Al-Boloushi Z, Gómez-Trullén EM, Bellosta-López P, López-Royo MP, Fernández D, Herrero P. Comparing two dry needling interventions for plantar heel pain: A protocol for a randomized controlled trial. J Orthop Surg Res. 2019;14(1):31. DOI: 10.1186/s13018-019-1066-4.

16. Hu HT, Gao H, Ma RJ, Zhao XF, Tian HF, Li L. Is dry needling effective for low back pain? Medicine. 2018;97(26):e11225. DOI: 10.1097/ MD.0000000000011225.

17. Dommerholt J, de las Peñas CF. Trigger Point Dry Needling E-Book: An Evidence and Clinical-Based Approach; 2013.

18. Dunning J, Butts R, Mourad F, Young I, Flannagan S, Perreault T. Dry needling: a literature review with implications for clinical practice guidelines. Phys Ther Rev. 2014;19(4):252-65. DOI: 10.1179/108331913X1 3844245102034.

19. Mayoral del Mayoral O, Salvat Salvat I. Fisioterapia Invasiva del Síndrome de Dolor Miofascial. Madrid: Panamerica; 2017.

20. Starkweather A. The Evidence on Dry Needling for Pain Management. Top Pain Manag. 2018;34(4):1-9. DOI: 10.2147/JPR.S154728.

21. Liberati A, Altman DG, Tetzlaff J, Mulrow C, Gøtzsche PC, loannidis JPA, et al. The PRISMA Statement for Reporting Systematic Reviews and Meta-Analyses of Studies That Evaluate Health Care Interventions: Explanation and Elaboration. PLoS Med. 2009;6(7):e1000100. DOI: 10.1371/journal.pmed.1000100.

22. Stewart LA, Clarke M, Rovers M, Riley RD, Simmonds M, Stewart G, et al. Preferred Reporting Items for a Systematic Review and Meta-analysis of Individual Participant Data. JAMA. 2015;313(16):1657-65. DOI: 10.1001/jama.2015.3656.

23. Harris RP, Helfand M, Woolf SH, Lohr KN, Mulrow CD, Teutsch SM, et al. Current methods of the US Preventive Services Task Force: a review of the process. Am J Prev Med. 2001;20(3 Suppl):21-35. DOI: 10.1016/ s0749-3797(01)00261-6.

24. Cotchett MP, Munteanu SE, Landorf KB. Effectiveness of Trigger Point Dry Needling for Plantar Heel Pain: A Randomized Controlled Trial. Phys Ther. 2014;94(8):1083-94. DOI: 10.2522/ptj.20130255.

25. Eftekharsadat B, Babaei-Ghazani A, Zeinolabedinzadeh V. Dry needling in patients with chronic heel pain due to plantar fasciitis: A single-blinded randomized clinical trial. Med J Islam Repub Iran. 2016;30:401.

26. El Mallah R, Elattar E, Zidan H. Platelet-rich plasma versus dry needling of myofascial meridian trigger points in the treatment of plantar fasciitis. Egypt Rheumatol Rehabil. 2017;44(2):58. DOI: 10.4103/1110161X.205661.

27. Lalchhuanawma A, Sen S, Dhawan A. Effectiveness of dry needling along with conventional therapy on myofascial trigger points in patients with plantar fasciitis. Int J Ther Rehabil Res. 2017;6(1):162-8. DOI: $10.2147 / J P R . S 141607$.

28. Rastegar S, Baradaran Mahdavi S, Hoseinzadeh B, Badiei S. Comparison of dry needling and steroid injection in the treatment of plantar fasciitis: a single-blind randomized clinical trial. Int Orthop. 2018;42(1):109-16. DOI: 10.1007/s00264-017-3681-1.

29. Rahbar M, Eslamian F, Toopchizadeh V, Jahanjoo F, Kargar A, Dolatkhah N. A Comparison of the Efficacy of Dry-Needling and Extracorporeal Shockwave Therapy for Plantar Fasciitis: A Randomized Clinical Trial. Iran Red Crescent Med J. 2018;20(9):e68908. DOI: 10.5812/ircmj.68908.

30. Uygur E, Aktaş B, Eceviz E, Yilmazoğlu EG, Poyanli O. Preliminary Report on the Role of Dry Needling Versus Corticosteroid Injection, an Effective Treatment Method for Plantar Fasciitis: A Randomized Controlled Trial. J Foot Ankle Surg. 2019;58(2):301-5. DOI: 10.1053/j.jfas.2018.08.058. 